\title{
Set-based parameter estimation for symmetric network motifs
}

\author{
Philipp Rumschinski ${ }^{* * *}$ Dina Shona Laila ${ }^{* *}$ Rolf Findeisen* \\ * Institute for Automation Engineering, Otto-von-Guericke University, \\ Magdeburg. \\ ** International Max Planck Research School, Magdeburg. \\ *** Kingston University and Imperial College, London. \\ e-mail: \{philipp.rumschinski,rolf.findeisen\}@ovgu.de, d.laila@ic.ac.uk
}

\begin{abstract}
Deriving a predictive model in systems biology is a complex task. One major problem is the typically large network size, which renders the analysis with standard methods difficult. Symmetry, as omnipresent in nature, was used in many applications to encounter this problem. In this work, we investigate the influence of symmetry on set-based parameter estimation. We show that the presence of symmetry in a model can be used to significantly simplify the parameter estimation problem. This is done by determining a symmetry-adapted basis, corresponding to a linear representation of a finite group, in which the problem size is of smaller dimension. We demonstrate the applicability of this approach for several common network motifs, as e.g. the Michaelis-Menten reaction and the feedforward motif.
\end{abstract}

Keywords: Parameter Estimation, Systems Biology, Symmetry

\section{INTRODUCTION}

Finding a model that can be used not only to describe but also to predict the behavior of a dynamical system under various initial conditions or external disturbances is a topic of almost every branch of science. Especially in systems biology, there is an increasing need for predictive models for instance to find treatments for diseases and to gain better understanding of complex biological processes.

Unfortunately, there are several issues that complicate the investigation of biological processes with existing standard analysis tools from systems and control theory. To mention a few, the quality of the available experimental data is often inadequate due to the presence of noise in concentration measurements or due to limited amount of data points extracted from invasive or destructive procedures [Ramsay et al., 2007]. In addition, biological systems typically exhibit a certain robustness against internal variations (e.g. random mutations), and external disturbances (e.g. environmental changes) [van Riel and Sontag, 2006]. These obstacles have been the stumbling block in identification process for the construction of a good quantitative model of biological processes. Several hypotheses would describe the measurements as it is often not possible to prove analytically the validity of the obtained nonlinear model. Instead, solving the converse problem, i.e. model invalidation, seems to be more promising in this situation and it has attracted a lot of attention in recent research [Anderson and Papachristodoulou, 2009, Melykuti et al., 2010]

A quite recent approach capable of giving conclusive answers to the model invalidation was presented in [Borchers et al., 2009, Rumschinski et al., 2010a]. The main idea of this approach is to relax a corresponding feasibility problem into a semi-definite program which can be solved efficiently. In addition to the model invalidation problem this relaxation provides a possibility to derive an outerapproximation of the parameter that leads to a consistent behavior of the model with the measurements. For this, the model invalidation is carried out for partitions of the parameter space, and it is then proved for parameter regions that cannot lead to a consistent behavior by a special class of infeasibility certificate. However, semidefinite programs still have a limited maximal problem size, which consequently poses a limit to the applicability of the aforementioned approach.

Symmetry has been successfully utilized in some earlier works to reduce the size of semi-definite optimization problems [Gatermann and Parrilo, 2004, Jansson et al., 2006]. These works did not try to circumvent the symmetry (symmetry breaking), but to use the symmetry of the model to reduce the problem size instead.

In this work we investigate the applicability of these approaches for set-based parameter estimation. We focus mainly on two arising problems. First, to examine the problem of finding a minimal symmetry-adapted basis for the optimization problem. This is done by determining the canonical decomposition of the linear representation of a group. Second, to show that knowledge about symmetries can also be used for finding optimization directions, that lead to good estimation results. We demonstrate the usefulness of this investigation using some simple network motifs.

Mathematical Notation: We denote with $g(x) \in \mathbf{G}$ a group-action defined for the group $\mathbf{G}$, with $x \in \mathbf{G}$. Aut and $\mathbf{D}$ denote the group of automorphisms under composition of maps and the dihedral group, respectively. 
$\mathcal{S}^{n_{M}} \subset \mathbb{R}^{n_{M} \times n_{M}}$ denotes the space of symmetric positivedefinite matrices, i.e. $M \in \mathcal{S}^{n_{M}} \Leftrightarrow M \succeq 0, M M^{T}=I$. $A \geq 0$ denotes the set of linear constraints given by the elements $a_{i j} \geq 0,0<i, j \leq n_{A}$ of $\mathrm{A}$. Index sets are denoted by $\mathcal{I}_{A}:=\left\{1, \ldots, n_{A}\right\}$ with $n_{A}$ corresponding to the dimension of the variable $A$. $\oplus$ denotes the direct-sum of vector spaces.

\section{PROBLEM SETUP}

Given a biochemical reaction network of the form

$$
\begin{aligned}
& \dot{x}=f(x, u, p) \\
& y=h(x, u, p),
\end{aligned}
$$

where $x \in \mathcal{X} \subseteq \mathbb{R}^{n_{x}}$ denotes the states (concentrations), $u \in \mathbb{R}^{n_{u}}$ the input (stimulus), $p \in \mathcal{P} \subseteq \mathbb{R}^{n_{p}}$ the system parameters (reaction constants) and $f, h$ are smooth polynomial functions.

Assume that at certain time instances $t_{i} \in \mathbb{R}_{>0}$ input and output measurements are taken. We denote with $\mathcal{T}=$ $\left\{t_{i}, i \in \mathcal{I} \subseteq \mathbb{Z} \mid a \leq t_{i} \leq b\right\}$ the set of all time instances each measurement was taken and $\mathcal{I}=\left\{1, \ldots, n_{\mathcal{I}}\right\}$ the set of time indices. Without loss of generality we assume that the initial condition is determined by the first measurement. The collections of input and output measurements are given respectively by

$$
\mathcal{U}=\left\{\mathcal{U}_{i} \subseteq \mathbb{R}^{n_{u}}, i \in \mathcal{I}\right\}, \quad \mathcal{Y}=\left\{\mathcal{Y}_{i} \subseteq \mathbb{R}^{n_{y}}, i \in \mathcal{I}\right\},
$$

where $\mathcal{U}_{i}, \mathcal{Y}_{i}$ are assumed to be uncertain, but bounded compact and convex sets, which allow a direct consideration of measurement errors. We assume further that $u$ is constant between sampling times.

Definition 1. (Model Consistency). Given the collection $\mathcal{U}$ and $\mathcal{Y}$ of all the measurements of inputs $u(\cdot)$ and output $y(\cdot)$, respectively. Model (1) is said to be consistent with the measurements, if for all $t_{i} \in \mathcal{T}$ it holds that $u\left(t_{i}\right) \in \mathcal{U}_{i}$ and $y\left(t_{i}\right) \in \mathcal{Y}_{i}$.

Accordingly, the set of parameters $\mathcal{P}^{*}$, that leads to a consistent behavior of model (1) is given by

$$
\begin{aligned}
\mathcal{P}^{*}=\left\{p \in \mathcal{P} \mid u\left(t_{i}\right) \in \mathcal{U}_{i}, y\left(t_{i}\right) \in \mathcal{Y}_{i}, \forall t_{i} \in \mathcal{T}:\right. \\
\dot{x}=f(x, u, p), y=h(x, u, p)\} .
\end{aligned}
$$

In [Borchers et al., 2009, Rumschinski et al., 2010a] a procedure was introduced to approximate $\mathcal{P}^{*}$ with the help of semi-definite programming for discrete-time models. For an approximation $\widehat{\mathcal{P}}$, to ensure that

$$
\mathcal{P}^{*} \subseteq \widehat{\mathcal{P}}
$$

holds, sufficient conditions were derived in [Rumschinski et al., 2010b]. In the remainder of this paper, it is therefore assumed that an appropriate discrete-time model of the form

$$
\begin{aligned}
x_{k+1} & =F_{T}\left(x_{k}, u_{k}, p\right) \\
y_{k} & =H_{T}\left(x_{k}, u_{k}, p\right)
\end{aligned}
$$

was chosen such that (4) holds.

Furthermore, we assume that the sampling period $T>0$ was chosen such that for every $t_{i} \in \mathcal{T}$ there exists $k \in \mathbb{N}$ such that $k T=t_{i}$. Then $T$ and $\mathcal{T}$ define implicitly the time index set $\mathcal{Z}$.

As it is known, the applicability of the set-based parameter estimation to large-scale systems suffers from its considerably high computational demand. In this work we present an extension based on a decomposition of the semi-definite program into smaller problems, which in many cases will reduce the computational effort drastically. This extension is based on the work of [Gatermann and Parrilo, 2004], [Jansson et al., 2006] and [Vallentin, 2009], and aims at building a simple algorithm to derive outer-bounds of the parameters. We, therefore, investigate the influence of symmetry in network motifs and the implications if such motifs are combined to form larger networks.

For this purpose we state here a rather general definition of symmetry and provide the more specific definition in later sections as needed.

Definition 2. Model (1) is called symmetric if there exists a mapping $\varphi: \mathcal{X} \times \mathcal{U} \times \mathcal{P} \rightarrow \mathcal{X} \times \mathcal{U} \times \mathcal{P}$ such that the set of consistent parameters $\mathcal{P}^{*}$ is invariant under $\varphi$, i.e.

$$
\begin{aligned}
\mathcal{P}^{*} \equiv\{p \in \mathcal{P} \mid x \in \mathcal{X}, u \in \mathcal{U}, y \in \mathcal{Y}: \\
\\
\dot{x}=f(\varphi(x, u, p)), y=h(\varphi(x, u, p))\} .
\end{aligned}
$$

\section{SYMMETRY IN SET-BASED PARAMETER ESTIMATION}

In this section we first summarize the set-based parameter estimation approach presented in [Borchers et al., 2009] and then derive a simplified version of this problem by exploiting symmetry of finite groups.

\subsection{Set-based Parameter Estimation: Review}

Suppose the experimental data $\mathcal{U}, \mathcal{Y}$ and a candidate model (5) are given. It is possible to gather all this information within the following set of (semi-)algebraic equations

$$
F(\mathcal{P}):\left\{\begin{array}{l}
x_{k+1}-F_{T}\left(x_{k}, u_{k}, p\right)=0 \\
y_{k}-H_{T}\left(x_{k}, u_{k}, p\right)=0 \\
p \in \mathcal{P} \\
x_{k} \in \mathcal{X} \\
u_{k} \in \mathcal{U} \\
y_{k} \in \mathcal{Y} \\
k \in \mathcal{Z}
\end{array}\right.
$$

Checking if $F(\mathcal{P})$ admits a solution or not, which we also denote as a feasibility problem, is equivalent to checking whether or not the model is able to reproduce the measurements for the given parameter set $\mathcal{P}$.

For the considered class of systems, we can address $F(\mathcal{P})$ in a relaxed form, namely as a semidefinite program $(S D P)$. Several methods for reformulating $F(\mathcal{P})$ in such a way are known in literature. For instance in [Lasserre, 2001], the problem is reformulated in terms of moments, which lead to a $S D P$ formulation with beneficial theoretical properties. However, this method might lead to rather large $S D P$ s if higher order moments are considered. Therefore, we restrict our investigation to a quadratic formulation following [Parrilo, 2003].

The basic idea here is to express every equation of the vector functions $x_{k+1}-F_{T}\left(x_{k}, u_{k}, p\right)=0$ and $y_{k}-$ $H_{T}\left(x_{k}, u_{k}, p\right)=0$ in a quadratic (second order) form $\xi^{T} Q_{k}^{j} \xi=0, j \in \mathcal{I}_{F}:=\left\{1, \ldots, n_{x}+n_{y}\right\}$ using a minimal monomial basis $\xi$. If $\xi$ contains monomials with order higher than 2, we introduce additional constraints in the 
form of $\xi^{T} Q_{k}^{j} \xi=0, j \in \mathcal{I}_{d}:=\left\{1, \ldots, n_{d}\right\}$, where $n_{d}$ corresponds to the number of lower order monomials necessary to decompose the higher order monomials into quadratic form. For shorthand notation we define $\mathcal{I}:=$ $\mathcal{I}_{F} \cup \mathcal{I}_{d}$.

Problem $F(\mathcal{P})$ can then subsequently be relaxed into a convex $S D P$ by introducing $X=\xi \cdot \xi^{T}$ and replacing the resulting conditions $\operatorname{rank}(X)=1$ and $\operatorname{tr}(X) \geq 1$ with the weaker constraint $X \succeq 0$, resulting in the relaxed formulation

$$
S D P(\mathcal{P}):\left\{\begin{aligned}
\min & \langle C, X\rangle \in \mathcal{S}^{n_{\xi}} \text { subject to } \\
& \left\langle Q_{k}^{j} X\right\rangle=0, \quad k \in \mathcal{Z}, j \in \mathcal{I}, \\
& e_{1} e_{1}^{T} X=1, \\
& A X e_{1} \geq 0, \\
& A X A^{T} \geq 0, \\
& X \succeq 0 .
\end{aligned}\right.
$$

where $e_{1}=(1,0, \ldots, 0)^{T} \in \mathbb{R}^{n_{\xi}}$. The constraint $A X e_{1} \geq 0$ corresponds to the bounds derived from the convex sets $\mathcal{X}, \mathcal{P}$ and the measurement data $\mathcal{U}, \mathcal{Y}$.

This leads to the following result:

Lemma 1. [Borchers et al., 2010] Given the measurement collections $\mathcal{U}, \mathcal{Y}$. If $S D P(\mathcal{P})$ is infeasible, then so is $F(\mathcal{P})$, i.e. model $(5)$ is inconsistent with the measurements.

Remark 2. As the relaxation process is conservative, each feasible solution for $F(\mathcal{P})$ corresponds to a feasible solution for $S D P(\mathcal{P})$. However, additional solutions might be introduced, which are only feasible for $S D P(\mathcal{P})$, but not for $F(\mathcal{P})$. The constraints $A X A^{T} \geq 0$ strengthen the relaxation and reduce this problem (e.g. [Lasserre, 2001], [Anstreicher, 2009]).

Note that $S D P(P)$ is formulated as an optimization problem in contrast to $F(\mathcal{P})$. This is derived from the fact that we want to get a good outer-approximation of the consistent parameter region as shown in the next section.

\subsection{Outer-Approximation of Consistent Parameters}

Given a direction $v \in \mathbb{R}^{n_{\xi}}$ and a corresponding cost $C_{v} \in \mathcal{S}^{n_{\xi}}$ for which $\left\langle C_{v}, X\right\rangle=v^{T} \xi$ holds, the result of (7) is a lower bound for the set of consistent parameters that is normal to $v$ (cf. Figure 1). Clearly, we obtain an upper bound by optimizing $\left\langle-C_{v}, X\right\rangle$.

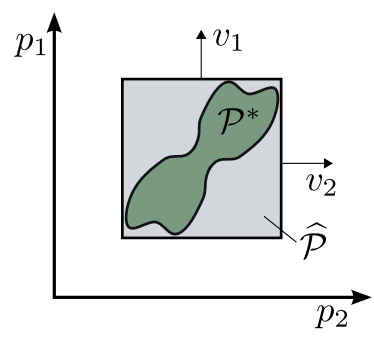

Fig. 1. Outer-approximation of consistent parameters

The estimated parameter region $\widehat{\mathcal{P}}$ is then given by the hyperplanes through the minimum (maximum) value derived by (7) orthogonal to $v_{i}$.

This simple scheme is well suited to obtain an outerapproximation of the consistent parameter region. However, the obtained bounds might be conservative in the sense that the consistent parameters are not oriented along the optimization direction.

It is hence immediately clear that the choice of directions $v$ is critical for the achievable quality of the outerapproximation. Without additional knowledge of the solution space of $S D P(\mathcal{P})$ choosing $v$ is very difficult. We propose in the following sections an approach for finding a different basis in which the feasible region can be better approximated. For further techniques of approximating the feasible region, we refer to [Borchers et al., 2010].

\subsection{Exploiting symmetry}

In this section we derive a symmetry-based reduction of $S D P(\mathcal{P})$ following [Gatermann and Parrilo, 2004, Vallentin, 2009]. The main idea employed here is the fact that every invariant matrix can be block-diagonalized by an appropriate change of coordinates. We start with some definitions and statements of representation theory.

We call a matrix $X \in S^{n_{\xi}}$ a feasible solution of (7) if it fulfills all constraints of (7). The $S D P(7)$ is invariant under the group $\mathbf{G}$ if for every feasible $X$ and for every $\varphi: \mathbf{G} \rightarrow \operatorname{Aut}\left(\mathcal{S}^{n_{\xi}}\right)$ the matrix $\varphi(X)$ is again a solution of (7). This corresponds to an a priori restriction of the solution space to a fixed-point subspace $\mathcal{V}$. In fact, if $X$ is an optimal solution of (7), so is its group average $\frac{1}{|G|} \sum_{\varphi \in \mathbf{G}} \varphi(X)$ as shown in [Gatermann and Parrilo, 2004]. Hence, (7) is equivalent to

$$
S D P(\mathcal{P}):\left\{\begin{array}{l}
\min \langle C, X\rangle \in \mathcal{S}^{n_{\xi}} \text { subject to } \\
\left\langle Q_{k}^{j} X\right\rangle=0, \quad k \in \mathcal{Z}, j \in \mathcal{I}, \\
e_{1} e_{1}^{T} X=1, \\
A X e_{1} \geq 0, \\
A X A^{T} \geq 0, \\
X \succeq 0 \\
X \in \mathcal{V} .
\end{array}\right.
$$

The fixed-point subspace $\mathcal{V}$ can be determined by constructing the linear representation of the group G. Note that for a finite group $\mathbf{G}$ only finitely many invariant representations $V_{1}, \ldots, V_{h}$ of dimensions $m_{1}, \ldots, m_{h}$ exist [Serre, 1977], where $m_{i}$ are divisors of the order of $\mathbf{G}$, i.e. $\bmod \left(|\mathbf{G}| / m_{i}\right)=0$. A canonical decomposition of the linear representation of $\mathbf{G}$ is given by

$$
m_{1} V_{1} \oplus m_{2} V_{2} \oplus \cdots \oplus m_{h} V_{h},
$$

with $m_{i} \in \mathbb{R}$ and $i \in \mathcal{I}_{h}:=\{1, \ldots, h\}$. After determining the symmetry-adapted basis (9), by setting $X=$ $\sum_{i=1}^{h} m_{i} V_{i}$ we can simplify (8) into

$$
\underline{S D P}(\mathcal{P}): \begin{cases}\min \sum_{i=1}^{h} m_{i}\left\langle C, V_{i}\right\rangle & \text { subject to } \\ \sum_{i=1}^{h} m_{i}\left\langle\bar{Q}_{k}^{j}, V_{i}\right\rangle=0, & k \in \mathcal{Z}, j \in \mathcal{I} \\ e_{1, i} e_{1, i}^{T} V_{i}=1, & i \in \mathcal{I}_{h}, \\ A_{i} V_{i} e_{1} \geq 0, & i \in \mathcal{I}_{h}, \\ A_{i} V_{i} A_{i}^{T} \geq 0, & i \in \mathcal{I}_{h}, \\ \sum_{i=1}^{h} m_{i} V_{i} \succeq 0, & \end{cases}
$$


where $e_{1, i}=(1,0, \ldots 0) \in \mathbb{R}^{n_{V_{i}}}, \bar{Q}_{k}^{j}$ and $A_{i}$ correspond to the orthogonal projection $Q_{k}^{j} \perp V_{i}$ and $A \perp V_{i}$, respectively.

Because $\mathcal{V}$ is closed under matrix multiplication, we know as a consequence of [Wedderburn, 1907] that there exists a unitary matrix $T$ that, by using Schur's Lemma $T^{-1} X T$, transforms $X$ into block-diagonal form. Note that since $T$ is unitary, the inverse matrix $T^{-1}$ is equal to the complex conjugate transpose of $T$. This block-diagonal structure can be exploited effectively by all commonly used interior point algorithms for solving an $S D P$.

Even if $X$ is a real symmetric matrix, the final blockdiagonal matrix might be complex as $T$ is allowed to be unitary. From a practical point of view, we are only interested in finding real transformations such that $T \in$ $\mathbb{R}^{n_{\xi} \times n_{\xi}}$ holds, $V_{i}$ has to be determined accordingly. A suitable algorithm was presented in [Serre, 1977]. However, if no such $T$ is available one can use the following relation

$$
X_{i} \succeq 0 \Leftrightarrow\left[\begin{array}{ll}
\mathfrak{R e}\left(X_{i}\right) & \mathfrak{I m}\left(X_{i}\right)^{T} \\
\mathfrak{I m}\left(X_{i}\right) & \mathfrak{R e}\left(X_{i}\right)
\end{array}\right] \succeq 0,
$$

to reformulate $S D P$ in terms of real matrices for every block $X_{i}$ in $T^{-1} X T$ ([Klerk, 2010]). Note that this reformulation doubles the size of each block $X_{i}$.

If we now choose $\varphi$ such that $\varphi(X)=T^{-1} X T$, (7) is simplified to the block-diagonalized $\underline{\overline{S D P}}(\mathcal{P})$ of the form

$$
\underline{\underline{S D P}}(\mathcal{P}): \begin{cases}\min \sum_{i=1}^{h} m_{i}\left\langle C, V_{i}\right\rangle & \text { subject to } \\ \sum_{i=1}^{h} m_{i}\left\langle\bar{Q}_{k}^{j}, V_{i}\right\rangle=0, & k \in \mathcal{Z}, j \in \mathcal{I} \\ e_{1, i} e_{1, i}^{T} V_{i}=1, & i \in \mathcal{I}_{h}, \\ A_{i} V_{i} e_{1} \geq 0, & i \in \mathcal{I}_{h}, \\ A_{i} V_{i} A_{i}^{T} \geq 0, & i \in \mathcal{I}_{h}, \\ \sum_{i=1}^{h} m_{i} \varphi\left(V_{i}\right) \succeq 0 . & \end{cases}
$$

Theorem 3. (Equivalency). If (7) is invariant with respect to $\varphi: \mathbf{G} \rightarrow \operatorname{Aut}\left(\mathcal{S}^{n_{\xi}}\right)$, the optimal solution $X^{*}$ of $(12)$ is equal to the optimal solution of (7).

Proof. The first direction is trivially given by the fact that $\varphi(X)$ is a solution of (7) as $X$ is invariant and we have $X^{*} \leq \varphi\left(X^{*}\right)$, the other direction is more involved and we refer to [Gatermann and Parrilo, 2004] for more details. $\square$

We also have the clear implication:

Corollary 4. (Model Invalidity). Given the measurement collections $\mathcal{U}, \mathcal{Y}$. If one of the blocks $X_{i}$ is infeasible, model (5) is inconsistent with the measurements.

Proof. Follows from the invariance of $X$.

In summary, we have shown that the parameter estimation problem can be formulated in terms of a symmetryadapted basis. This has the clear advantage in which (12) has a structure that is easier to solve and will typically have a smaller size of problem as the sum $m_{1}+\ldots+m_{h}$ is smaller than the dimension of $X$.

However, in practice we have several issues to consider. Computing the basis $V_{i}$ and therefore $\varphi$ can be difficult as it relies typically on randomized algorithms [Vallentin, 2009]. Furthermore, the sum of squares problem investigated in [Gatermann and Parrilo, 2004] is different from the problem we are investigating. For instance, as the states are typically concentrations, that are positive, a symmetry based on the change of sign is not possible. Further, if additional constraints - for instance monotonicity constraints - are considered, it may result in breaking the symmetry [Anstreicher, 2009]. This results in the fact that in biochemical reaction networks symmetries concerning the states are not as relevant.

However, despite the aforementioned problems that may pose a limitation, we are mainly concerned with the parameter estimation. We investigate the symmetry properties only in the parameters (rather than the states) and the influence of such symmetries to the region of consistent parameters. Hence, the main advantage is not the reduction in size but the reduction in the volume of the outerapproximation of the region of consistent parameters.

\subsection{Symmetry-adapted Outer-approximation}

In the previous section we have shown that for symmetric systems we can address the parameter estimation in new coordinates. As depicted in Fig. 2 this can lead to an outer-approximation $\widehat{\mathcal{P}}$ that approximates the consistent parameters more closely.

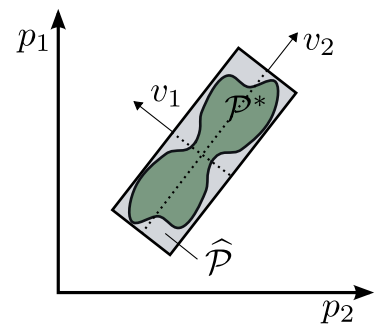

Fig. 2. Outer-approximation of consistent parameters with symmetry adapted basis vectors $v_{i}$.

We provide next several examples of symmetric network motifs, which show that the symmetry-adapted parameter estimation formulation delivers tighter results than the standard one.

\section{EXAMPLES: SYMMETRY IN NETWORK MOTIFS}

In this section we investigate the symmetry properties of some simple network motifs described in [Alon, 2007]. For simplicity of presentation, we will consider for all example systems the same computational setup and the same group, namely the dihedral group D. Furthermore, we assume that all states are measured.

Computational Setup: The simulation studies were conducted by generating first a nominal trajectory $x^{*}$ for each model with the nominal parameter values $p_{i}^{*}=1$ $(i \in\{1,2\}, i \in\{1,2,3\}, i \in\{1,2,3,4\}$ depending on the considered problem). As the initial condition of the appearing concentrations we have chosen $A_{0}=0.8, B_{0}=$ $0.1, C_{0}=0.1, D_{0}=0.1$. For the numerical integration of the models we have used ode23s in MATLAB. For the 
measurements at every $k \in\{1, \ldots, 40\}$ and a time stepsize $T=0.5 \mathrm{~s}$ a measurement was created by $\mathcal{Y}_{k}=\left\{x_{k} \in\right.$ $\left.\mathbb{R}^{4}:\left\|x_{k}-x^{*}(k T)\right\| \leq 0.1\right\}$. As an initial parameter set we assume for each $p_{i}$ the interval $[0.33,3]$. The setbased parameter estimation was implemented on a standard desktop computer, using YALMIP and a parallelized version of the solver CSDP.

\subsection{Linear Motif}

Consider a simple reaction motif

$$
A \underset{p_{2}}{\stackrel{p_{1}}{\rightleftharpoons}} B
$$

with $p_{i}$ denoting the constant reaction rates. The corresponding mass-action equations are given by

$$
\begin{aligned}
& \frac{d A}{d t}=-p_{1} A+p_{2} B, \\
& \frac{d B}{d t}=p_{1} A-p_{2} B .
\end{aligned}
$$

Obviously, this reaction is symmetric to the representations $\varphi_{1}:\left(p_{1}, p_{2}\right) \rightarrow\left(p_{2}, p_{1}\right)$ and $\varphi_{2}:\left(p_{1}, p_{2}\right) \rightarrow\left(-p_{2}, p_{1}\right)$, which corresponds to interchanging the species $A, B$ and reversing the reactions, respectively. This implies that if the dihedral group $\mathbf{D}_{3}$ is considered, we have the following symmetry-adapted basis corresponding to the 2 dimensional irreducible representations of $\mathbf{D}_{3}$

$$
V_{1}=\left[\begin{array}{cc}
-\frac{1}{2} & \frac{\sqrt{3}}{2} \\
-\frac{\sqrt{3}}{2} & -\frac{1}{2}
\end{array}\right], V_{2}=\left[\begin{array}{cc}
1 & 0 \\
0 & -1
\end{array}\right]
$$

Results: The parameters were once estimated by means of the axis-aligned parameter estimation and once with the symmetry-adapted basis. The results are shown in Fig. 3. The symmetry-adapted estimates were obtained in roughly $17 \mathrm{~s}$ (22s in the non-adapted basis).

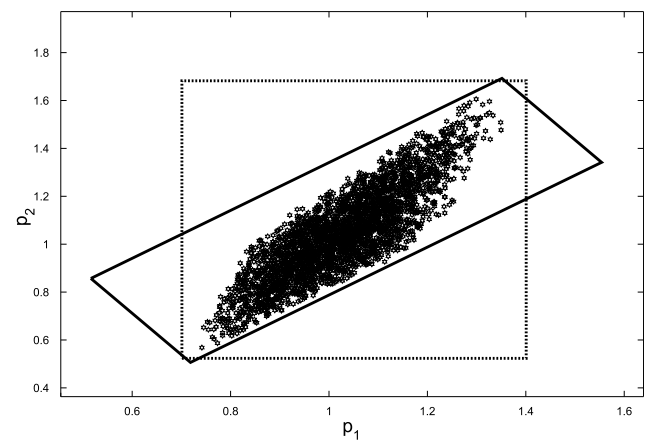

Fig. 3. Outer-approximation of consistent parameters for model (13). Dotted lines axis aligned box, full line symmetry adapted box, dots correspond to 3000 Monte Carlo samples.

\subsection{Enzyme-catalyzed Reaction}

Consider the motif

$$
A+B \underset{p_{2}}{\stackrel{p_{1}}{\rightleftharpoons}} C \stackrel{p_{3}}{\rightarrow} D+B .
$$

Here a substrate $A$ joins an enzyme $B$ to form the complex $C$, and finally the product $D$ is released. Note that this motif represents the Michaelis-Menten reaction. If we consider the conservation relation $B+C=1$, we get the following model equations

$$
\begin{aligned}
& \frac{d A}{d t}=p_{1} A B-p_{1} A+p_{2} B \\
& \frac{d B}{d t}=p_{1} A-p_{1} A B-\left(p_{2}+p_{3}\right) B .
\end{aligned}
$$

Results: The estimates, as depicted in Fig. 4, were obtained in roughly $41 \mathrm{~s}$ (49s in the non-adapted basis). Note that the volume decreases for the adapted case noticeably.

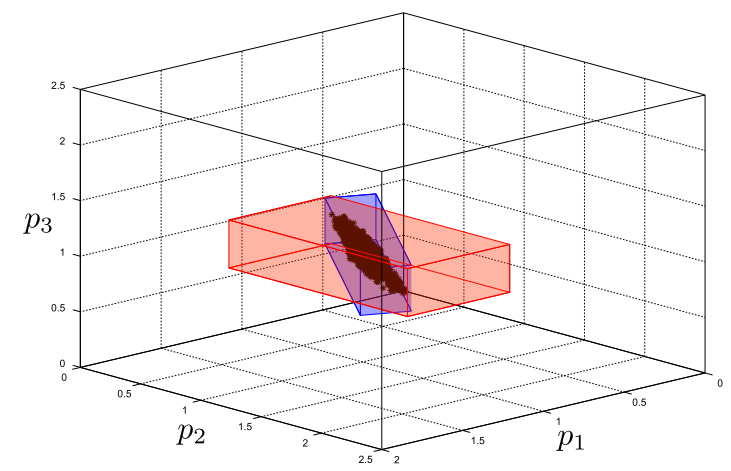

Fig. 4. Outer-approximation of consistent parameters for model (16). Red lines axis aligned box, blue line symmetry adapted box, dots correspond to 1500 Monte Carlo samples.

\subsection{Feedforward and Feedback Motifs}

As one of the most frequent network motifs, we have the feedforward motif.

$$
A \stackrel{p_{3}}{\stackrel{p_{1}}{\rightleftharpoons}} \stackrel{p_{4}}{\searrow} B
$$

Note that this also corresponds to a feedback motif, if the arrow orientation is changed. This motif, using massaction kinetics, can be described by

$$
\begin{aligned}
& \frac{d A}{d t}=-p_{1} A-p_{3} A+p_{2} B, \\
& \frac{d B}{d t}=p_{1} A-p_{2} B+p_{4} C, \\
& \frac{d C}{d t}=p_{3} A-p_{4} C .
\end{aligned}
$$

Results: The results are shown in Table 1 and Table 2 . The estimates for the symmetry-adapted basis were obtained in roughly $228 \mathrm{~s}$ (272s in the non-adapted basis). The content (hypervolume) is 0.046 (0.065 in the nonadapted basis).

Interestingly, the decrease in volume allows in the two directions with the symmetry for a better approximation of the remaining directions. Note that the increase in the parameter values results from the fact that by transforming the edges back into the axis oriented coordinates result in a certain conservatism (cf. direction of $p_{1}$ in Fig. 3). 
Table 1. Consistent parameter region using symmetry-adapted basis.

\begin{tabular}{c|cccc}
\hline & $p_{1}$ & $p_{2}$ & $p_{3}$ & $p_{4}$ \\
\hline lower Bound & 0.7093 & 0.6857 & 0.8156 & 0.7481 \\
\hline upper Bound & 1.3053 & 1.3644 & 1.2150 & 1.3066 \\
\hline \hline
\end{tabular}

Table 2. Consistent parameter region using the non-adapted basis.

\begin{tabular}{c|cccc}
\hline & $p_{1}$ & $p_{2}$ & $p_{3}$ & $p_{4}$ \\
\hline lower Bound & 0.7642 & 0.7604 & 0.8023 & 0.7336 \\
\hline upper Bound & 1.2407 & 1.2962 & 1.2322 & 1.3228 \\
\hline \hline
\end{tabular}

\section{CONCLUSIONS}

In this work we have proposed an extension to the setbased parameter estimation and model invalidation approach presented in [Borchers et al., 2009, Rumschinski et al., 2010a]. This method is based on the solution of a relaxed version of a nonlinear feasibility problem, which provides conclusive answers to the model invalidation problem. Furthermore, it allows to derive an outerapproximation of the consistent parameters. A major challenge that has to be considered when applying this approach is the computational tractability. Therefore, we have investigated the applicability of a reduction procedure proposed in [Gatermann and Parrilo, 2004] for symmetric sum-of-square problems.

We have shown that this concept of symmetry can also be used to reduce the computational effort necessary for set-based parameter estimation. This was done by limiting the optimization problem directly to the fixedpoint subspace of the solution space corresponding to the symmetry. Therefore, we have derived the canonical decomposition of linear representation of a finite group following [Gatermann and Parrilo, 2004] and [Vallentin, 2009]. We have pointed out that in systems biology, this method may not be applied directly due to the problem structure that is to some extent different. However, as the examples suggest a consideration of symmetry only in the parameters allows still to obtain a better outerapproximation of the consistent parameters compared to when symmetry is not considered.

A further reduction of the computational effort could be achieved by limiting the monomial basis of the semidefinite program to the invariant polynomial ring. This would reduce not only the problem size, but also limit the amount of algebraic operations necessary for solving the optimization as shown in [Gatermann and Parrilo, 2004].

\section{ACKNOWLEDGEMENTS}

We thank our colleague Anton Savchenko for providing an efficient implementation of the set-based parameter estimation. This work was supported in part by the Federal Ministry of Education and Research (BMBF) in Germany, under the Forsys-Partner-Project (grant no. 0315-280D).

\section{REFERENCES}

U. Alon. An introduction to systems biology. Chapman \& Hall/CRC, Boca Raton, FL, 2007.

J. Anderson and A. Papachristodoulou. On validation and invalidation of biological models. BMC Bioinformatics, 10(1):132, 2009.

K.M. Anstreicher. Semidefinite programming versus the reformulation-linearization technique for nonconvex quadratically constrained quadratic programming. Journal of Global Optimization, 43(2):471-484, 2009.

S. Borchers, P. Rumschinski, S.Bosio, R. Weismantel, and R. Findeisen. A set-based framework for coherent model invalidation and parameter estimation of discrete time nonlinear systems. In Proc. IEEE Conf. on Dec. and Contr., CDC '09, pages 6786-6792, Shanghai, China, 2009.

S. Borchers, S. Bosio, P. Rumschinski, A. Savchenko, R. Weismantel, and R. Findeisen. Guaranteed estimation, classication, and analysis of polynomial systems: A relaxation-based framework. Automatica, 2010. Submitted.

K. Gatermann and P.A. Parrilo. Symmetry groups, semidefinite programs, and sums of squares. J. Pure and Appl. Algebra, 192(1-3):95-128, 2004.

L. Jansson, J.B. Lasserre, C. Riener, and T. Theobald. Exploiting symmetries in SDP-relaxations for polynomial optimization. Opt. Online, 2006.

E. Klerk. Exploiting special structure in semidefinite programming: a survey of theory and applications. Euro. J. of Oper. Res., 201(1):1-10, 2010.

J.B. Lasserre. Global optimization with polynomials and the problem of moments. SIAM J. on Opt., 11(3):796817, 2001.

B. Melykuti, E. August, A. Papachristodoulou, and H. ElSamad. Discriminating between rival biochemical network models: three approaches to optimal experiment design. BMC Syst. Biol., 4(1):38, 2010.

P.A. Parrilo. Semidefinite programming relaxations for semialgebraic problems. Math. Progr., 96(2):293-320, 2003.

J.O. Ramsay, G. Hooker, D. Campbell, and J. Cao. Parameter estimation for differential equations: A generalized smoothing approach. J. R. Stat. Soc.-Ser. B, 69:741796, 2007.

P. Rumschinski, S. Borchers, S. Bosio, R. Weismantel, and R. Findeisen. Set-based dynamical parameter estimation and model invalidation for biochemical reaction networks. BMC Syst. Biol., 4:69-82, 2010a.

P. Rumschinski, D. S. Laila, and R. Findeisen. Influence of discretization errors on set-based parameter estimation. In Proc. IEEE Conf. on Dec. and Contr., CDC '10, 2010b. To appear.

J.P. Serre. Linear representations of finite groups. Springer Verlag, 1977.

F. Vallentin. Symmetry in semidefinite programs. Lin. Algebra and its Appl., 430(1):360-369, 2009.

N.A.W. van Riel and E.D. Sontag. Parameter estimation in models combining signal transduction and metabolic pathways: the dependent input approach. Syst. Biol., 153:263-274, 2006.

J.H.M. Wedderburn. On hypercomplex numbers. Proc. London Math. Soc., 6(2):77-118, 1907. 